\title{
ECDC guidance on prevention of HIV and STIs suggests seven components for inclusion in national EU/ EEA public health programmes
}

On 17 June, the European Centre of Disease Prevention and Control published an evidence-based guidance document on the prevention of human immunodeficiency virus (HIV) and sexually transmitted diseases (STIs) among men who have sex with men (MSM) [1]. The guidance lists seven key components i.e. vaccinations, condoms, HIV and STI testing, treatment, health promotion, MSM-competent health services and targeted care for MSM living with HIV that should be considered for inclusion in the public health programmes of European Union (EU) and European Economic Area (EEA) countries. The guidance elaborates on:

- Promotion and delivery of vaccination to protect against hepatitis $A$ and $B$. Consideration of vaccination for human papilloma virus.

- Provision of easily accessible condoms and condom-compatible lubricants and promotion their effective use.

- Provision of voluntary and confidential HIV and STI counselling and testing via a variety of modalities that are easy to access for the target group. Voluntary partner referral can support the early diagnosis and treatment of contacts.

- Timely provision of treatment for HIV, viral hepatitis and STI should be ensured. Significance of preventive benefits of treatment.

- Provision of accurate and accessible information that enables men to understand and assess sexual health-related risks and prevention efficacy, and that promotes awareness of one's own HIV and STI status.

- MSM-competent points of care offering a comprehensive sexual health programme including health promotion, counselling, peer support, prevention, adequate diagnostics and treatment will increase service uptake. Ensure target group involvement and training for providers on how to offer comprehensive care for MSM.
- Provide antiretroviral treatment for HIV and vaccination; regular STI screening using adequate diagnostics; treatment for STIs; individual counselling, sexual health promotion and peer-support groups for men living with HIV.

The suggestions are based on the opinion of an expert panel and on a systematic review, published in Eurosurveillance earlier this year [2].

Men who have sex with men are disproportionately affected by HIV and other STIs including gonorrhoea, syphilis, chlamydia and hepatitis B and C. This pattern is the same in all EU/EEA countries.

\section{References}

1. European Centre for Disease Prevention and Control (ECDC). HIV and STI prevention among men who have sex with men. Stockholm: ECDC; 2015. Available from: http://ecdc.europa. eu/en/publications/layouts/forms/Publication DispForm. aspx?List=4f55ad51-4aed-4d32-b960-af70113dbb9o\&ID=1319

2. Strömdahl S, Hickson F, Pharris A, Sabido M, Baral S, Thorson A. A systematic review of evidence to inform HIV prevention interventions among men who have sex with men in Europe. Euro Surveill. 2015;20(15):pii=21096. Available from: http:// www.eurosurveillance.org/ViewArticle.aspx?Articleld=21096 